\title{
Both Sm-domain and C-terminal extension of Lsm1 are important for the RNA-binding activity of the Lsm1-7-Pat1 complex
}

\author{
ASHIS CHOWDHURY, KALIDINDI K. RAJU, ${ }^{1}$ SWATHI KALURUPALLE, and SUNDARESAN THARUN ${ }^{2}$ \\ Department of Biochemistry, Uniformed Services University of the Health Sciences (USUHS), Bethesda, Maryland 20814-4799, USA
}

\begin{abstract}
Lsm proteins are a ubiquitous family of proteins characterized by the Sm-domain. They exist as hexa- or heptameric RNAbinding complexes and carry out RNA-related functions. The Sm-domain is thought to be sufficient for the RNA-binding activity of these proteins. The highly conserved eukaryotic Lsm1 through Lsm7 proteins are part of the cytoplasmic Lsm1-7-Pat1 complex, which is an activator of decapping in the conserved $5^{\prime}-3^{\prime}$ mRNA decay pathway. This complex also protects mRNA 3 '-ends from trimming in vivo. Purified Lsm1-7-Pat1 complex is able to bind RNA in vitro and exhibits a unique binding preference for oligoadenylated RNA (over polyadenylated and unadenylated RNA). Lsm1 is a key subunit that determines the RNA-binding properties of this complex. The normal RNA-binding activity of this complex is crucial for mRNA decay and 3 '-end protection in vivo and requires the intact Sm-domain of Lsm1. Here, we show that though necessary, the Sm-domain of Lsm1 is not sufficient for the normal RNA-binding ability of the Lsm1-7-Pat1 complex. Deletion of the C-terminal domain (CTD) of Lsm1 (while keeping the Sm-domain intact) impairs mRNA decay in vivo and results in Lsm1-7-Pat1 complexes that are severely impaired in RNA binding in vitro. Interestingly, the mRNA decay and 3' -end protection defects of such CTD-truncated Ism1 mutants could be suppressed in trans by overexpression of the CTD polypeptide. Thus, unlike most Sm-like proteins, Lsm1 uniquely requires both its Sm-domain and CTD for its normal RNA-binding function.
\end{abstract}

Keywords: Lsm1-7-Pat1 complex; mRNA decay; Sm-like; decapping; Lsm1

\section{INTRODUCTION}

The Lsm proteins (also known as Sm-like proteins) are a ubiquitous family of proteins characterized by the presence of the Sm-domain. They typically exist as ring-shaped, six or seven-membered homo- or heteromeric complexes that bind RNA and carry out RNA-related functions (Beggs 2005; Wilusz and Wilusz 2005; Tharun 2009). The Smdomain is made up of two conserved blocks of residues (Sm-motifs I and II) separated by a nonconserved linker region of variable length (Cooper et al. 1995; Hermann et al. 1995; Seraphin 1995; Salgado-Garrido et al. 1999). The Sm-domain adopts a unique 3D structure ("Sm-fold") consisting of an $\mathrm{N}$-terminal $\alpha$-helix and a strongly bent

\footnotetext{
${ }^{1}$ Present address: Department of Biotechnology, Gokaraju Rangaraju Institute of Engineering and Technology (GRIET), Bachupally, Kukatpally, Hyderabad 500090, India.

${ }^{2}$ Corresponding author.

E-mail tsundaresan@usuhs.mil.

Article published online ahead of print. Article and publication date are at http://www.rnajournal.org/cgi/doi/10.1261/rna.029876.111.
}

5-stranded $\beta$-sheet (Kambach et al. 1999; Collins et al. 2001; Mura et al. 2001; Toro et al. 2001; Schumacher et al. 2002). Here, the RNA contacting residues are present in the loop regions 3 (between $\beta$-strands $\beta 2$ and $\beta 3$ ) and 5 (between $\beta$-strands $\beta 4$ and $\beta 5$ ) (Kambach et al. 1999; Mura et al. 2001; Toro et al. 2001; Mura et al. 2003a; Mikulecky et al. 2004). In general, the Sm-domain seems to be sufficient for the RNA binding and oligomerization activities of the Lsm proteins, since most Lsm proteins are small, such that the Sm-domain ( $\sim 80$ residues) occupies most of the polypeptide.

The spliceosomal Sm proteins and the Lsm1 through Lsm8 proteins are highly conserved eukaryotic members of the Lsm protein family. The Lsm1 through Lsm7 proteins bind to Pat1 and form the cytoplasmic Lsm1-7-Pat1 complex (Bouveret et al. 2000; Tharun et al. 2000; Tharun 2009; Totaro et al. 2011). This complex is required for normal rates of decapping in the conserved $5^{\prime}-3^{\prime}$ mRNA decay pathway, wherein decapping is a key step that is dependent on prior deadenylation and permits $5^{\prime}-3^{\prime}$ exonucleolytic degradation of the message body (Bouveret et al. 2000; 
Tharun et al. 2000; Coller and Parker 2004; Garneau et al. 2007; Houseley and Tollervey 2009; Tharun 2009). Lsm1 is a key subunit that distinguishes this complex from the nuclear localized Lsm2-8 complex (made of Lsm2 through Lsm8 proteins), which is not involved in cytoplasmic mRNA decay (Bouveret et al. 2000; Tharun et al. 2000; Ingelfinger et al. 2002; Tharun 2009). In addition to being an activator of decapping, Lsm1-7-Pat1 complex also protects the $3^{\prime}$-ends of mRNAs from trimming in vivo (Boeck et al. 1998; He and Parker 2001; Tharun et al. 2005). We showed earlier that the purified Lsm1-7-Pat1 complex is able to bind RNA in vitro and exhibits a strong binding preference for RNA substrates with oligo(A) tail (Chowdhury et al. 2007), and also showed that such RNA-binding properties of this complex are crucial for mRNA decay in vivo (Chowdhury and Tharun 2008). Mutagenic analyses revealed that the residues in loops 3 and 5 of the Smdomain of Lsm1 are critical determinants of the RNAbinding properties of the Lsm1-7-Pat1 complex (Tharun et al. 2005; Chowdhury and Tharun 2008).

Intriguingly, yeast Lsm 1 has extended $\mathrm{N}$ - and C-terminal domains ( $\sim 40$ and $\sim 55$ residues, respectively) flanking the $\mathrm{Sm}$-domain. While the $\mathrm{N}$-terminal domain is absent in human Lsm1 and functionally dispensable in yeast, the C-terminal domain (CTD) is necessary for both mRNA decay and $3^{\prime}$-end protection in yeast (Tharun et al. 2005). Consistently, human Lsm1 also carries a long CTD with moderate similarity to yeast Lsm1's CTD (Tharun et al. 2005). Nevertheless, the function of the CTD of Lsm1 is not known. Surprisingly, our results presented here reveal that the normal RNA-binding activity of the Lsm1-7-Pat1 complex requires not only the Sm-domain, but also the CTD of Lsm1, and show that the CTD is able to support the function of this complex even when it is not contiguous with the Sm-domain of Lsm1 (in trans). Thus, the Sm-domain is not sufficient for the RNA-binding activity of Lsm1, wherein the CTD also seems to be very important. These results imply that other Sm-like proteins with extended $\mathrm{N}$ - or C-terminal segments could also bear functionally critical regions capable of modulating the RNA-binding activity of the Sm-domain in those segments.

\section{RESULTS}

\section{CTD of Lsm1 is necessary for the normal RNA-binding activity of the Lsm1-7-Pat1 complex}

To understand the function of the CTD of yeast (Saccharomyces cerevisiae) Lsm1, we had created the $1 s m 1-27$, $1 s m 1-28$, and $l s m 1-29$ alleles that expressed truncated versions of Lsm1 lacking 55, 43, and 28 residues, respectively, from the $\mathrm{C}$ terminus, but carrying intact Sm-domain and N-terminal extension (i.e., residues 1-117) (Fig. 1A; Tharun et al. 2005). Analysis of the $M F A 2 p G$ reporter mRNA revealed that
(A)

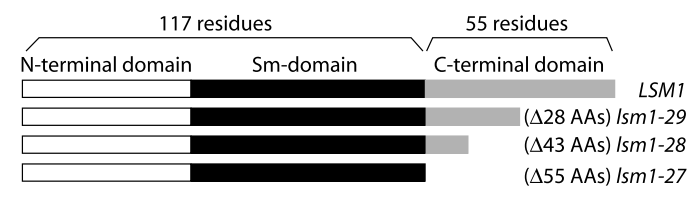

(B)
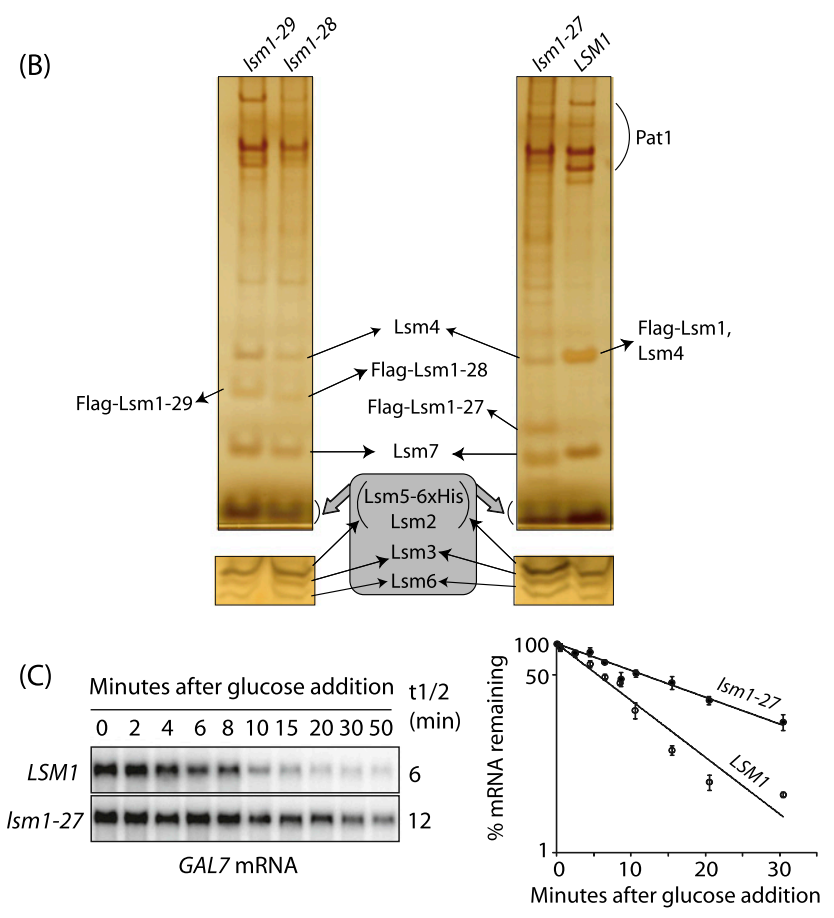

FIGURE 1. Truncation of the CTD of Lsml affects the decay of the endogenous GAL7 mRNA without abolishing the formation of the Lsm1-7-Pat1 complex. (A) Schematic diagram showing the extent of deletions in $l s m 1-27, l s m 1-28$, and $l s m 1-29$. (B) Purification of the mutant Lsm1-7-Pat1 complexes. Lsm1-7-Pat1 complexes were purified from the strains indicated on top, separated by SDS-PAGE, and silver stained (upper panels). The lower band resolves into three bands in a $16 \%$ gel, part of which is shown at the bottom. (C) The endogenous GAL7 mRNA is stabilized in 1 sm1-27 cells. Cells were grown to log phase in galactose medium to express the GAL7 mRNA, whose transcription is then shut off by shifting the cells to glucose medium. Following this, RNA was made from the cells at different time points and subjected to Northern analysis, followed by PhosphorImager quantitation of the bands to determine the rate of disappearance of the GAL7 mRNA. The level of this mRNA in each sample was normalized for that of 7S RNA of SRP (to serve as loading control), which was determined by reprobing the blot for that RNA. A phosphorimage of the blot and a plot of the amount of RNA remaining versus time after shifting cells to the glucose medium are shown on the left and right panels, respectively.

mRNA decay and $3^{\prime}$-end protection are impaired in $l s m 1 \Delta$ cells expressing any of these alleles from a $C E N$ vector using native promoter and UTR sequences (Tharun et al. 2005), and that such impairment is not affected by $\mathrm{N}$-terminal "FLAG"-tagging of these lsm 1 alleles (Figs. 1C, 4, below; Supplemental Figs. S1E, S2B). In order to confirm that the decay of endogenous mRNA is also impaired upon truncating the CTD of Lsm1, we measured the half-life of GAL7 and GAL10 mRNAs (whose transcription can be shut off by shifting the cells to glucose medium) in $1 s m 1-27$ and 
lsm1-28 mutants. We observed that both of these mRNAs are stabilized in these mutants compared with the wild-type cells (Fig. 1C; Supplemental Fig. S1E; data not shown). Western analysis revealed that the CTD-truncated mutant Lsm 1 proteins are expressed in the mutant cells at levels similar to that of wild-type Lsm1 in wild-type cells (Supplemental Fig. S1B). Purification of the truncated Lsm1 containing complexes from these mutants (using the strategy we described earlier) (Chowdhury et al. 2007; Tharun 2008), followed by comparison of the SDS-PAGE band patterns of the mutant and wild-type complexes and determination of the tryptic peptide sequences (by mass spectrometry analysis) of the proteins present in the purified mutant complexes revealed that the mutant complexes contain all of the expected subunits of the Lsm1-7-Pat1 complex (Fig. 1B). Thus, inability to assemble the complex is not the likely cause of the mRNA decay and $3^{\prime}$-end protection defects in these mutants.

The purified wild-type Lsm1-7-Pat1 complex binds RNA and exhibits an intrinsic preference for oligoadenylated RNA over unadenylated RNA in vitro (Chowdhury et al. 2007). Therefore, we studied the RNA-binding activity of the mutant complexes purified from $l s m 1-27, l s m 1-28$, and lsm1-29 cells in gel-shift assays using uniformly radiolabeled PGK1 and MFA2 RNAs (42-mer RNAs derived from the 3' UTRs of the yeast MFA2 and PGK1 genes, respectively) (Chowdhury et al. 2007; Chowdhury and Tharun 2008) that lack or carry a $3^{\prime}-\mathrm{A}_{5}$ tail. As seen in Figure $2 \mathrm{~A}$, in gelshift assays using MFA2 RNA, the mutant complexes exhibited lower RNA-binding ability than the wild-type complex with the Lsm1-27 and Lsm1-28 containing complexes exhibiting especially poor binding ability. Nevertheless, addition of an $\mathrm{A}_{5}$ tail enhanced binding of the MFA2 RNA to not only the wild-type, but also the mutant complexes. Similar results were obtained in analogous experiments using $P G K 1$ and $P G K 1-A_{5}$ RNAs (Fig. 2B,C). Thus, the truncation of the CTD of Lsm1 impairs the overall RNA-binding ability of the Lsm1-7Pat1 complex, but does not abolish the binding preference of the Lsm1-7-Pat1 complex for oligoadenylated RNA.

It is known that among unadenylated RNA substrates, the wild-type Lsm1-7-Pat1 complex has a strong binding preference for those that carry a U-tract at or near the $3^{\prime}$ end over those that do not (Chowdhury et al. 2007). Since the PGK1 RNA carries a 3 '-U8 tract, we tested the effect of replacing this $U 8$-tract with non- $U$ residues (GACACCAG; $P G K 1-U_{8} m u t$ RNA). Gel-shift assays using PGK1 and $P G K 1-U_{8} m u t$ RNAs revealed that the $P G K 1-U_{8} m u t$ RNA bound more weakly than the PGK1 RNA not only to the wild-type complex, but also to the Lsm1-27 and Lsm1-28 complexes (data not shown; Supplemental Fig. S1A). This suggests that truncation of the CTD of Lsm1 does not abolish the ability of the Lsm1-7-Pat1 complex to distinguish between RNAs with and without $3^{\prime}$-U-tracts.

\section{Primary sequence of the CTD of Lsm1 is critical for the function of the Lsm1-7-Pat1 complex}

Past studies on other Lsm proteins support the notion that the Sm-domain is sufficient for the RNA-binding activity of the Lsm proteins. Therefore, the above results are surprising since the Sm-domain is intact in the $l s m 1-27, l s m 1-28$, and $l s m 1-29$ alleles. Homology-based structure prediction using the Swiss-Model program (Kiefer et al. 2009) suggested that the folding of the Sm-domain of Lsm1 is not affected by the truncation of the CTD of Lsm1. To ensure that the observed functional consequences of CTD deletion are not due to any indirect effects of truncation of the Lsm1 polypeptide, we replaced the C-terminal 43 residues of S. cerevisiae Lsm1 with the corresponding region of human, Pichia stipitis or Candida glabrata Lsm1 ortholog generating the chimeric genes yyhLSM1, yyPsLSM1, and $y y C g L S M 1$, respectively. We expressed each of these alleles using the native yeast LSM1 promoter from a CEN vector in $l s m 1 \Delta$ cells and tested its ability to support mRNA decay and $3^{\prime}$-end protection in vivo using the $M F A 2 p G$ reporter mRNA. The $3^{\prime}$ UTR of this reporter mRNA carries a $\operatorname{poly}(\mathrm{G})$ insertion that blocks Xrn1 action in cis, such that the $5^{\prime}-3^{\prime}$ decay of this mRNA results in the accumulation of a decay intermediate called poly $(\mathrm{G})$ fragment (Decker and Parker 1993). The level of this fragment at steady-state is a good indicator of the efficiency of $5^{\prime}-3^{\prime}$ decay in vivo, because defects in decapping and/or $5^{\prime}-3^{\prime}$ exonucleolysis lead to a reduction of such levels (Hatfield et al. 1996; Tharun et al. 2005). Defects in $3^{\prime}$-end protection cause 3 '-end trimming of the $M F A 2 p G$ mRNA, and, therefore, also lead to the accumulation of a significant fraction of the poly $(G)$ fragment in trimmed form, so that the size of such fraction is a measure of the status of $3^{\prime}$-end protection in vivo (Tharun et al. 2005).

Northern analysis of the RNA made from $l s m 1 \Delta$ cells expressing the chimeric LSM1 genes revealed that the accumulation of the $\operatorname{poly}(\mathrm{G})$ fragment is increased and the fraction of the $\operatorname{poly}(\mathrm{G})$ fragment in trimmed form is decreased in cells expressing $y y C g L S M 1$ or $y y P s L S M 1$, but not yyhLSM1, compared with the untransformed $l s m 1 \Delta$ cells (Fig. 3A). Also, the accumulation of MFA2pG mRNA in deadenylated form (which is increased by defects in decapping or $5^{\prime}-3^{\prime}$ exonucleolysis) was decreased in cells expressing $y y C g L S M 1$ or $y y P S L S M 1$ compared with the untransformed $l s m 1 \Delta$ cells. Similar results were obtained using strains of a different genetic background (Supplemental Fig. S2A). Further, accumulation of the deadenylated form of the endogenous RPL41A mRNA was also decreased in $1 s m 1 \Delta$ cells expressing $y y C g L S M 1$ or $y y P s L S M 1$ (but not $y y h L S M 1$ ) compared with the untransformed $l s m 1 \Delta$ cells (Fig. 3B). Thus, the expression of $y y C g L S M 1$ or $y y P s L S M 1$, but not $y y h L S M 1$, causes a significant suppression of the mRNA decay and $3^{\prime}$-end protection defects in $l s m 1 \Delta$ cells. This observation is consistent with the CTD of human Lsm1 being less similar 
(A)
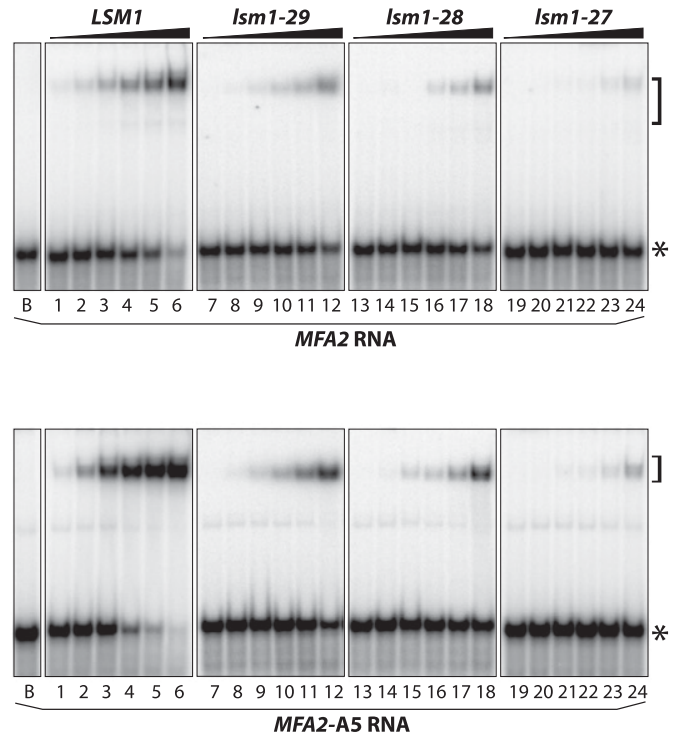

(B)
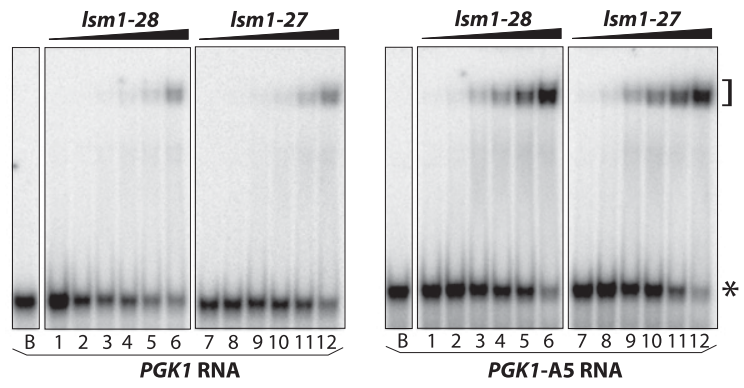

(C)

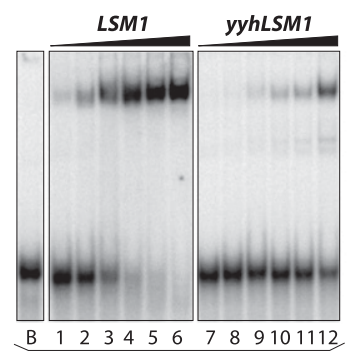

PGK1 RNA

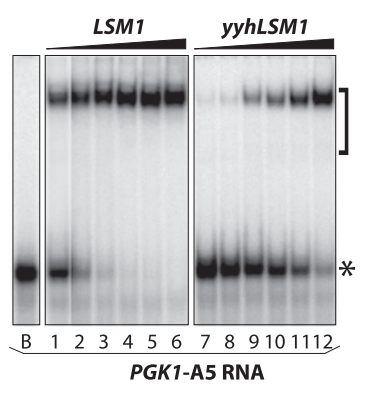

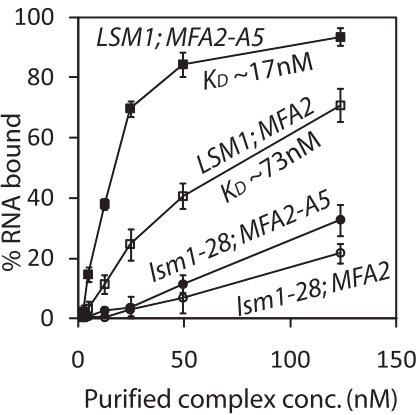
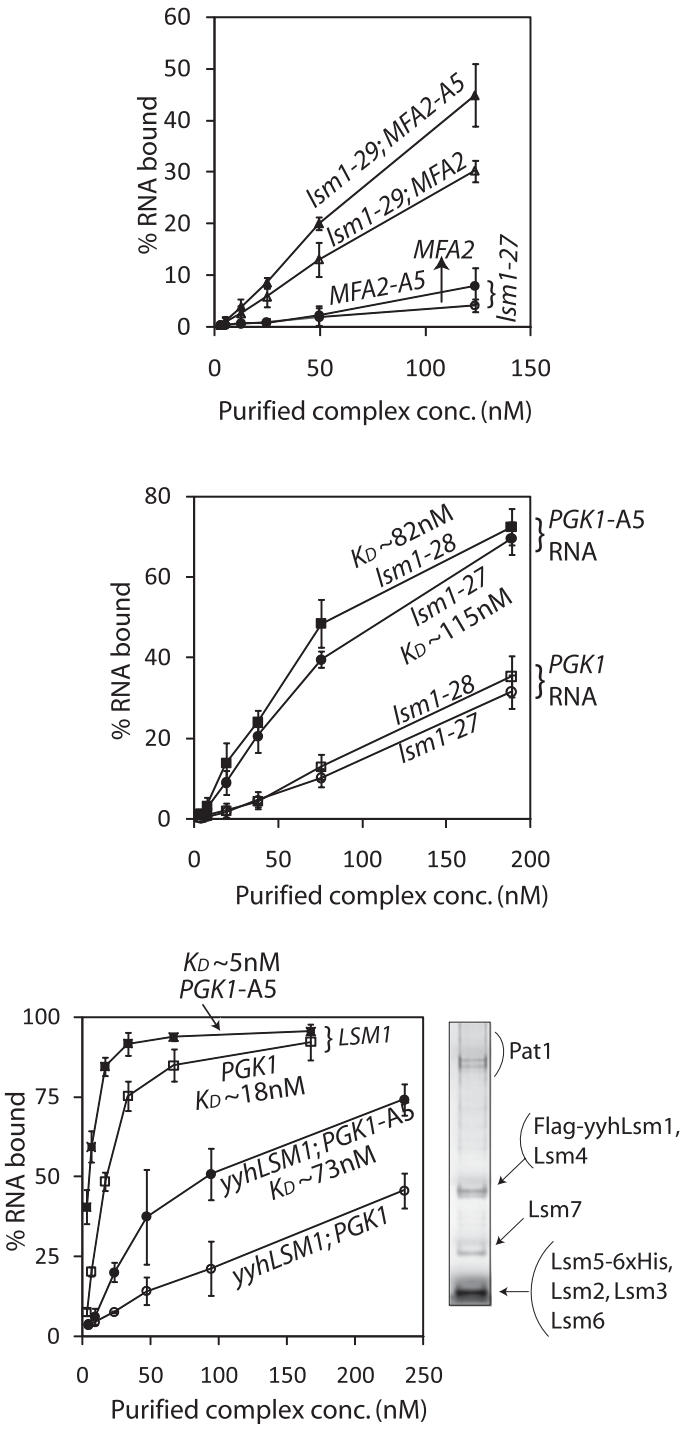

FIGURE 2. CTD of Lsm1 is necessary for the normal RNA-binding activity of the Lsm1-7-Pat1 complex. Increasing concentrations of Lsm1-7Patl complexes purified from the strains indicated on top (or BSA in lanes marked "B") were subjected to gel-shift assays using uniformly radiolabeled MFA2 and MFA2- $\mathrm{A}_{5}$ RNAs $(A)$ or PGK1 and PGK1- $\mathrm{A}_{5}$ RNAs $(B, C)$. Plots of \% RNA bound versus the concentration of the complex used are shown on the right of the phosphorimages of the gels. Bound and unbound RNA bands are indicated by brackets and asterisks, respectively, on the right. The faster moving bound RNA bands are probably due to the disassembly of the RNP complexes during the gel run. In $C$, the right panel shows SDS-PAGE separation of yyhLsm1 containing complex (silver stained).

than that of P. stipitis (or C. glabrata) Lsm1 to the CTD of S. cerevisiae Lsm1 (Supplemental Fig. S1C). This indicates that the primary sequence of the CTD of Lsml is crucial for the functioning of the Lsm1-7-Pat1 complex. Therefore, the CTD is a functionally critical segment of the Lsm1 polypeptide and the in vivo and in vitro functional defects 

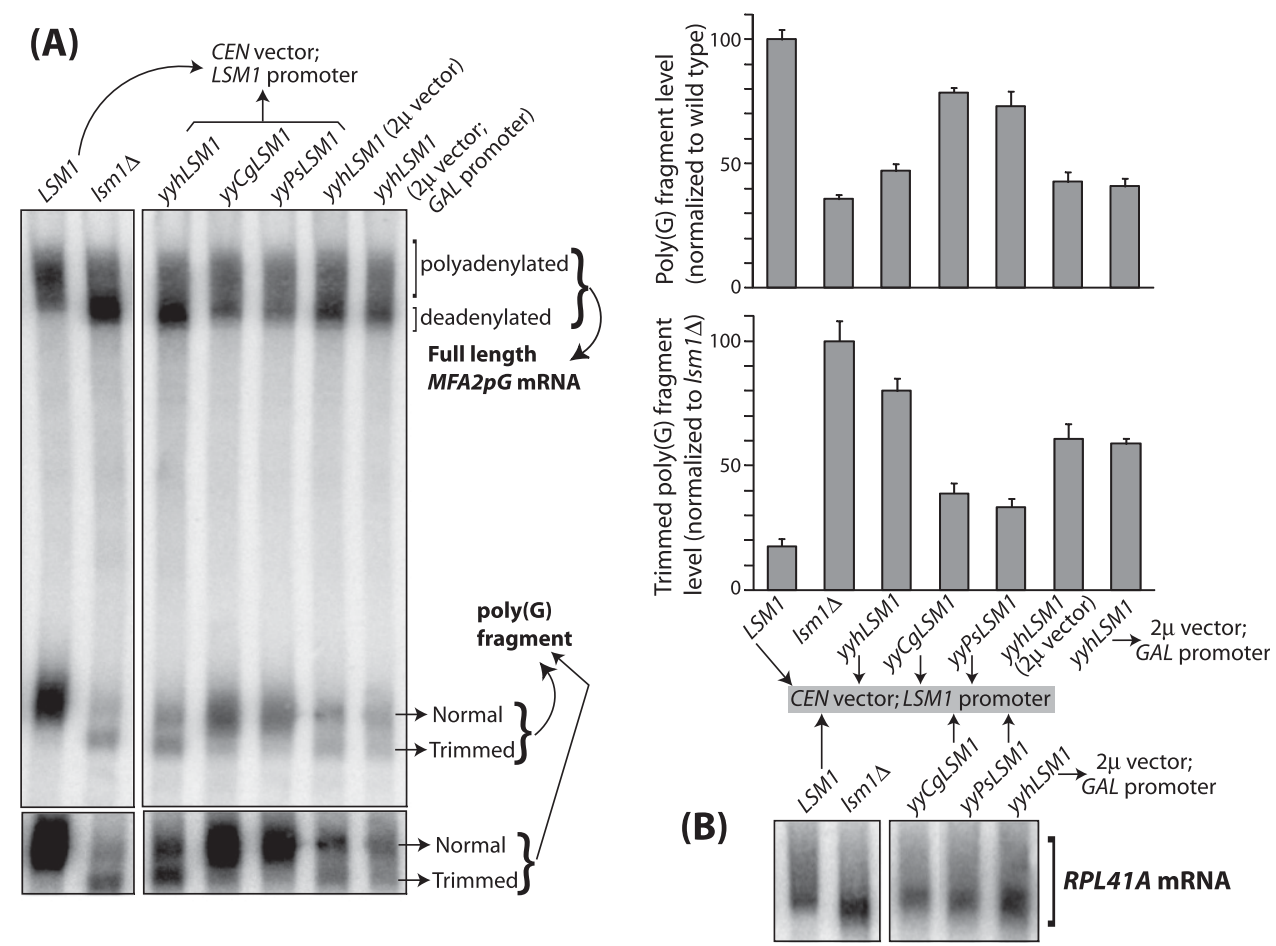

FIGURE 3. Primary sequence of the CTD is critical for Lsm1 function in vivo. (A) RNA isolated from lsmld strains expressing different LSM1 chimeras as indicated on top were subjected to Northern analysis to reveal the $M F A 2 p G$ mRNA and the poly $(\mathrm{G})$ fragments. A phosphorimage of the blot is shown in the top left panel. A longer exposure of the section of the blot containing the poly $(\mathrm{G})$ fragment bands is shown in the lower left panel. The fractional contribution of the poly $(G)$ fragments to the total signal (total: full-length mRNA + trimmed and normal poly $(G)$ fragments) was determined for each sample (following quantitation of the respective bands in the corresponding lane using the PhosPhorimager), normalized to the wild-type value and presented as a bar diagram in the top, right panel. Similarly, the fractional contribution of the trimmed poly $(\mathrm{G})$ fragment to the total poly $(\mathrm{G})$ fragment signal (trimmed + normal) was also determined for each sample, normalized to the value obtained for the $l s m 1 \Delta$ sample, and presented as a bar diagram in the bottom right panel. (B) RNA isolated from $l$ sm $1 \Delta$ strains expressing different LSM1 chimeras as indicated on top were subjected to Northern analysis to reveal the RPL41A mRNA.

pertaining to the $l s m 1-27, l s m 1-28$, and $l s m 1-29$ alleles are not just indirect effects of polypeptide truncation.

Inability of $y$ hLSM1 to suppress the mRNA decay defect of the $l s m 1 \Delta$ cells is not due to its insufficient expression, because the suppression was not significantly enhanced upon overexpression from high-copy $2 \mu$ vectors (using the yeast LSM1 promoter or the Gal promoter) (Fig. 3; Supplemental Figs. S1D, S2A). Nevertheless, electrophoretic and mass spectrometry analyses of the yyhLsm1-containing complex purified from cells that express the $y y h L S M 1$ allele using the yeast $L S M 1$ promoter from a $2 \mu$ vector revealed that it contains all of the subunits (Fig. 2C). Gel-shift assays revealed that similar to the Lsm1-27 or Lsm1-28-containing complex, the yyhLsm1-containing complex also has a lower RNA-binding ability than the wild-type complex, but retains the binding preference for oligoadenylated RNAs (Fig. 2C). Thus, in $l s m 1 \Delta$ cells expressing yyhLSM1, decapping may be blocked at a step after mRNA binding by the Lsm1-7-Pat1 complex.

\section{CTD of Lsm1 can function in trans}

The above results show that Lsm1 can fold and form the complex with other subunits, even after its CTD is deleted.
Therefore, we asked whether the CTD could complement the functional defects of the CTD-truncated $l s m 1$ alleles in trans. Indeed, study of the status of the MFA2pG mRNA and the poly $(\mathrm{G})$ fragments via Northern analysis revealed that expression of the C-terminal 60 residues of Lsm1 as a separate polypeptide at least partly suppresses the mRNA decay and $3^{\prime}$-end protection defects of the CTD-truncated $1 s m 1$ mutants. This was evident from an increase in the total poly $(\mathrm{G})$ fragment level and a decrease in the fraction of the poly $(\mathrm{G})$ fragment in trimmed form in the mutant cells expressing the CTD peptide compared with the cells that do not (Fig. 4A). Further, in the case of both MFA2pG mRNA (Fig. 4A) and the endogenous RPL41A mRNA (Fig. $4 \mathrm{~B}$ ), accumulation of deadenylated species observed in the CTD-truncated $l s m 1$ mutants was reduced by the expression of the CTD peptide. These results were reproducibly observed using strains of two different genetic backgrounds (Fig. 4; Supplemental S2B). Pull-down of the CTD peptide from these $1 s m 1-27$ and $1 s m 1-28$ cells coprecipitated the corresponding mutant Lsm1 proteins (Fig. 5A), suggesting that the observed phenotypic suppression indeed results from the association of the CTD peptide with the truncated Lsm1 proteins. Such association is unlikely to involve non- 
(A)
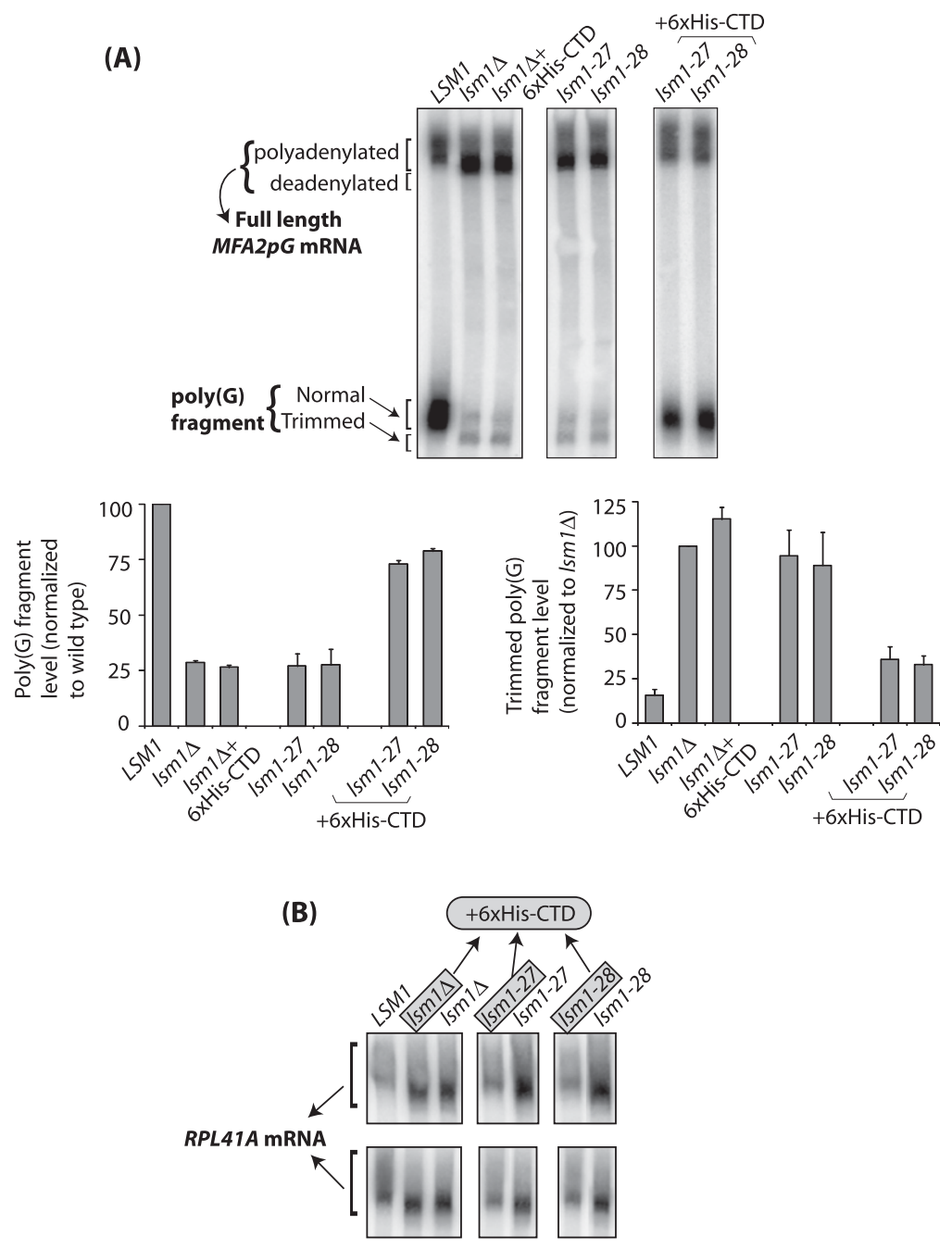

FIGURE 4. The 60-mer CTD polypeptide suppresses the mRNA decay and 3 '-end protection defects of the CTD-truncated lsm 1 alleles in trans. MFA2pG mRNA and the poly $(\mathrm{G})$ fragments $(A)$ or RPL41A mRNA $(B)$ present in the RNA isolated from the CTD-truncation mutants of lsm 1 (expressing or not expressing $6 \mathrm{x}$-His tagged CTD peptide) and control strains (indicated on top) were visualized by Northern analysis. A phoshorimage of the blot and bar diagrams showing the accumulation of the poly $(\mathrm{G})$ fragments (quantitated as described for Fig. 3 ) are shown in the top and bottom panels, respectively, in $A$. In $B$, the top and bottom panels show RNA from strains of two different strain backgrounds.

native interactions because expression of the CTD peptide did not significantly affect mRNA decay or $3^{\prime}$-end trimming in wild-type (data not shown) and $l s m 1 \Delta$ (Fig. 4) cells (as revealed by the analysis of MFA2pG and RPL41A mRNAs) and the pull-down of the CTD peptide from such wild-type cells did not coprecipitate the wild-type Lsm1 (Fig. 5A).

We then asked whether the CTD peptide could complement the RNA binding defect of the purified Lsm1-27 complex in vitro. The Lsm1-27 complex was immobilized onto the anti-FLAG antibody matrix before or after incubating it with a synthetic peptide containing the C-terminal 55 residues of yeast Lsm1. After washing, the matrix was incubated with uniformly radiolabeled PGK1 RNA and washed again. The bound RNA was then extracted and visualized by denaturing gel run and phosphorimaging. As seen in Figure 5B and Supplemental Fig. S2C, preincubation with the CTD peptide significantly enhanced RNA binding by the immobilized Lsm1-27 complex, but not the wild-type complex. Therefore, the CTD suppresses the mRNA decay and $3^{\prime}$-end protection defects of the CTD-truncated $l s m 1$ mutants in trans by virtue of its ability to associate with the mutant Lsm1-7-Pat1 complex, and thereby facilitate RNA binding by that complex.

\section{DISCUSSION}

The Lsm1-7-Pat1 complex is unique among the Sm-like protein complexes in being able to distinguish oligoadenylated RNA from unadenylated and polyadenylated RNAs. Such ability and the overall RNA-binding activity of this complex are crucial for mRNA decay and $3^{\prime}$-end protection in vivo and are critically dependent on the residues in the Sm-domain of the Lsm1 subunit (Tharun et al. 2005; Chowdhury and Tharun 2008). The key finding presented here is that the CTD of Lsm1 is also required (in addition to the Smdomain) for the normal RNA-binding activity of this complex. This explains the mRNA decay and 3 '-end protection defects of CTD-truncated $l s m 1$ mutants.

An important question raised by these studies is how the CTD of Lsm1 affects RNA binding by the Lsm1-7Pat1 complex. Our studies support the idea that the CTD acts primarily by facilitating RNA-binding activity of the Sm-domain of Lsm1 (or other subunits of the complex) rather than forming an additional RNA-binding surface of its own for the following reasons. Although the deletion of the CTD of Lsm1 results in a severe impairment of the RNAbinding activity of the Lsm1-7-Pat1 complex, it abolishes neither of the two important RNA-binding properties of this complex, namely, the strong binding preference for oligoadenylated RNAs over unadenylated RNAs and the binding preference for unadenylated RNAs carrying a U-tract near the $3^{\prime}$-end over those that do not (Chowdhury et al. 2007). Thus, the complexes purified from the CTDtruncated $l s m 1$ mutants, $l s m 1-27$ and $l s m 1-28$, exhibit weaker affinity (compared with the wild-type complex) 
(A)

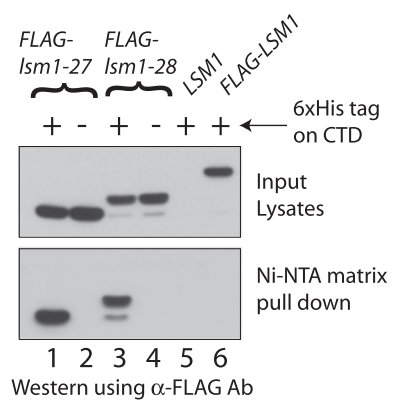

(B)

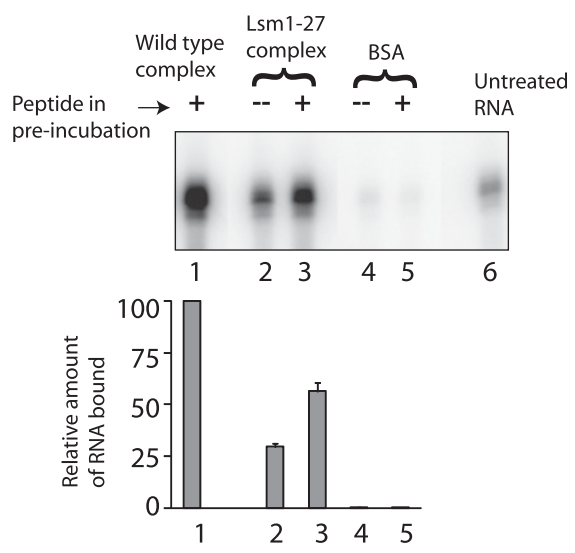

FIGURE 5. CTD peptide associates with the mutant Lsm1-7-Pat1 complex (containing CTD-truncated Lsm1) and enhances its RNAbinding activity. ( $A$ ) Lysates from strains (indicated on top) expressing untagged or His-tagged CTD polypeptide and proteins pulled down from such lysates using the Ni-NTA matrix were subjected to Western analysis using anti-FLAG antibodies. $(B)$ Purified wild-type and Lsm1-27 containing complexes or BSA were incubated with the antiFLAG antibody matrix (after or without preincubation with synthetic CTD peptide) and the ability of the immobilized material to bind radiolabeled PGK1 RNA was studied as described in the text. RNA bound to the matrix in each experiment was visualized on a denaturing gel (top) and its amount (quantitated using a PhosPhorimager) was shown as a bar diagram below the corresponding lane.

for all types of RNA substrates studied, i.e., RNAs with and without oligo(A) tail and unadenylated RNAs with and without 3'-U-tracts. However, importantly, the ability of the Lsm1-7-Pat1 complex to recognize the oligo(A) tail can be abolished by mutating the predicted RNA-binding pocket residues located in loops 3 or 5 (while keeping the CTD intact) of the Sm domain of Lsm1, such that the mutant complex from $1 s m 1-14$ cells exhibits normal affinity for unadenylated and polyadenylated RNAs, but reduced affinity for oligoadenylated RNAs, so that all of these RNA substrates are bound with comparable affinities (Chowdhury and Tharun 2008). This underscores the importance of the Sm domain of Lsm1 for such recognition. Further, some Sm-domain mutations of Lsm1 (in the presence of intact CTD) can result in almost complete loss of the RNAbinding ability of the Lsm1-7-Pat 1 complex (Chowdhury and Tharun 2009). Finally, the CTD of Lsm1 does not contain any recognizable motifs. In any case, we cannot completely rule out the possibility of some of the CTD residues contacting RNA directly, although our efforts to show direct binding of RNA by the CTD peptide has not been successful so far.

The observation that, in addition to the Sm-domain, the CTD of Lsm1 is also required for the normal in vivo functions of the Lsm1-7-Pat1 complex in mRNA decay and 3 '-end protection and for the normal RNA-binding activity of the purified Lsm1-7-Pat1 complex in vitro indicates that the scenario of Lsm1 is in contrast to many other Lsm family members wherein the $\mathrm{Sm}$-domain generally seems to be sufficient for function. Most Sm-like proteins are very small, such that the Sm-domain occupies almost the whole protein, although some Lsm family members do carry extended N- or C-terminal segments like Lsm1. For example, the Lsm4 subunit of the Lsm1-7-Pat1 complex has a C-terminal extension following its Sm-domain. However, deletion of this C-terminal segment does not affect mRNA decay in vivo (Decker et al. 2007), suggesting that it is unlikely to cause a significant impairment of the RNAbinding activity of the Lsm1-7-Pat1 complex. Similarly, the essential protein Lsm8, which is the key distinguishing subunit of the nuclear Lsm2-8 complex (that forms the core of the U6 snRNP), also carries a C-terminal extension following its Sm-domain. However, deletion of this C-terminal segment does not affect the viability in yeast, suggesting that it has a minimal effect on the splicing function of the U6 snRNP (Reijns et al. 2009). Nevertheless, the E. coli Hff protein presents a different picture. While Hfq's Sm-domain residues form two RNA-binding surfaces that bind small noncoding regulatory RNAs (ncRNAs) and poly(A), the CTD residues form a third RNA-binding surface that binds mRNAs, so that deletion of the CTD does not affect ncRNA or poly(A) binding and affects only mRNA binding (Mikulecky et al. 2004; Vecerek et al. 2008). This is in contrast to the CTD of Lsm1, which, as revealed by our studies, is necessary for the overall RNA-binding activity of the Lsm1-7-Pat1 complex, i.e., it is required for binding RNAs with and without oligo(A) tail and unadenylated RNAs with and without $3^{\prime}$-U-tracts with wild-type affinities.

Our pull-down experiments show that the CTD peptide expressed in trans associates with the CTD-truncated mutant Lsm1 proteins (Lsm1-27 and Lsm1-28), but not with the wild-type Lsm1. This implies that the CTD of Lsm1 makes specific contacts with one or more sites on the Lsm17-Pat1 complex. At present we do not know which subunits carry such sites. Structural studies on the SmAP3 (archaeal Lsm protein) homoheptamer and the homooctamer formed by yeast Lsm3 in bacteria reveal that the residues in the C-terminal extension could facilitate intersubunit interactions within the Lsm ring and between two Lsm rings (Mura et al. 2003b; Naidoo et al. 2008). These observations suggest that the CTD of Lsm1 may also make contacts with the Lsm2 through Lsm7 or Pat1 subunits. In any case, the CTD of 
Lsm1 is not required for the Lsm1-7Pat1 complex formation, since our results show that both Lsm1-27 (which lacks the entire CTD) and yyhLsm1 (wherein the CTD is replaced with human Lsm1's CTD, which is functionally deficient in the context of the yeast Lsm1) are able to form the Lsm1-7Pat1 complex. Thus, the interactions of the CTD of Lsm1 (with the Sm domain of Lsm1 or with other subunits), though important functionally, do not seem to be necessary for the integrity of the Lsm1-7-Pat1 complex. These results are consistent with studies on other Smlike proteins, which also show that the Sm-domain is sufficient for oligomerization. For example, although human Sm-B and Sm-D3 proteins carry long CTDs, their CTDs are dispensable for B-D3 subcomplex formation (Hermann et al. 1995; Kambach et al. 1999).

Apart from Lsm1, another subunit that is likely to be very critical for the Lsm1-7-Pat1 complex function is Pat1. pat1s mutant has a strong mRNA decay and 3 '-end protection phenotypes like the lsm1s mutant (Bonnerot et al. 2000; Bouveret et al. 2000; Tharun et al. 2000). Yeast Pat1 can associate with poly(U) sepharose, suggesting that it is an RNA-binding protein (Pilkington and Parker 2008). However, the contribution of Pat 1 to the RNA binding and other functions of Lsm17-Pat1 complex is not known. It is possible that Pat 1 facilitates the RNA binding by the Lsm1-7-Pat1 complex by providing an additional RNA-binding surface of its own.

Overall, these studies suggest that polypeptide segments that modulate the RNA-binding functions of the Smdomain could occur in the $\mathrm{N}$ - and C-terminal extensions (regions that flank the Sm-domain) of Sm-like proteins, and therefore have important implications to the biology of this large ubiquitous family of proteins.

\section{MATERIALS AND METHODS}

Strains needed for Lsm1-7-Pat1 complex purifications were made by introducing the plasmids expressing different $l s m 1$ alleles into yST247 (Chowdhury et al. 2007), which is in the genetic background of yRP841 (Hatfield et al. 1996). Strains used for the experiments shown in Figures 1C, 3B, 4A,B (top) and Supplemental Figures S1 and $\mathrm{S} 2 \mathrm{~A}$, are also in this background and were generated by transforming the $l s m 1 \Delta$ strain yRP1365 (Tharun et al. 2000) with appropriate plasmids. Strains used for experiments shown in Figures $3 \mathrm{~A}$ and $4 \mathrm{~B}$ (bottom) and Supplemental Figure S2B were made by transforming yST188 (trp1A1, his3-11,-15, ura3-1, leu2-3,-112, ade2-1, can1-100, lsm1s::TRP1, [pGal-MFA2pG]), which is in the genetic background of BMA64 (Mayes et al. 1999). Plasmids used in this study are described in Table 1.
Purification of Lsm1-7-Pat1 complex, preparation of radiolabeled RNAs, gel-shift assays, mRNA stability measurement and Northern, pull-down, and Western analyses were done as described (Chowdhury et al. 2007; Chowdhury and Tharun 2008; Chowdhury and Tharun 2009).

\section{SUPPLEMENTAL MATERIAL}

Supplemental material is available for this article.

\section{ACKNOWLEDGMENTS}

This work was supported by funds from NIH RO1 grant (GM072718) and USUHS exploratory grant (RO71JX) to S.T. We thank Dr. Roy Parker for his comments on the manuscript.

Received August 13, 2011; accepted February 24, 2012.

\section{REFERENCES}

Beggs JD. 2005. Lsm proteins and RNA processing. Biochem Soc Trans 33: 433-438.

Boeck R, Lapeyre B, Brown CE, Sachs AB. 1998. Capped mRNA degradation intermediates accumulate in the yeast $s p b 8-2$ mutant. Mol Cell Biol 18: 5062-5072.

Bonnerot C, Boeck R, Lapeyre B. 2000. The two proteins Patlp (Mrtlp) and Spb8p interact in vivo, are required for mRNA decay, and are functionally linked to Pablp. Mol Cell Biol 20: 5939-5946.

Bouveret E, Rigaut G, Shevchenko A, Wilm M, Seraphin B. 2000. A Sm-like protein complex that participates in mRNA degradation. EMBO J 19: 1661-1671.

Chowdhury A, Tharun S. 2008. lsm 1 mutations impairing the ability of the Lsm1p-7p-Patlp complex to preferentially bind to oligoadenylated RNA affect mRNA decay in vivo. RNA 14: 2149-2158. 
Chowdhury A, Tharun S. 2009. Activation of decapping involves binding of the mRNA and facilitation of the post-binding steps by the Lsm1-7-Pat1 complex. RNA 15: 1837-1848.

Chowdhury A, Mukhopadhyay J, Tharun S. 2007. The decapping activator Lsm1p-7p-Patlp complex has the intrinsic ability to distinguish between oligoadenylated and polyadenylated RNAs. RNA 13: 998-1016.

Coller J, Parker R. 2004. Eukaryotic mRNA decapping. Annu Rev Biochem 73: 861-890.

Collins BM, Harrop SJ, Kornfeld GD, Dawes IW, Curmi PM, Mabbutt BC. 2001. Crystal structure of a heptameric Sm-like protein complex from archaea: implications for the structure and evolution of snRNPs. J Mol Biol 309: 915-923.

Cooper M, Johnston LH, Beggs JD. 1995. Identification and characterization of Uss1p (Sdb23p): a novel U6 snRNA-associated protein with significant similarity to core proteins of small nuclear ribonucleoproteins. EMBO J 14: 2066-2075.

Decker CJ, Parker R. 1993. A turnover pathway for both stable and unstable mRNAs in yeast: Evidence for a requirement for deadenylation. Genes Dev 7: 1632-1643.

Decker CJ, Teixeira D, Parker R. 2007. Edc3p and a glutamine/ asparagine-rich domain of Lsm4p function in processing body assembly in Saccharomyces cerevisiae. J Cell Biol 179: 437-449.

Garneau NL, Wilusz J, Wilusz CJ. 2007. The highways and byways of mRNA decay. Nat Rev Mol Cell Biol 8: 113-126.

Hatfield L, Beelman CA, Stevens A, Parker R. 1996. Mutations in transacting factors affecting mRNA decapping in Saccharomyces cerevisiae. Mol Cell Biol 16: 5830-5838.

He W, Parker R. 2001. The yeast cytoplasmic LsmI/Patlp complex protects mRNA 3' termini from partial degradation. Genetics 158: $1445-1455$.

Hermann H, Fabrizio P, Raker VA, Foulaki K, Hornig H, Brahms H, Luhrmann R. 1995. snRNP Sm proteins share two evolutionarily conserved sequence motifs which are involved in Sm proteinprotein interactions. EMBO J 14: 2076-2088.

Houseley J, Tollervey D. 2009. The many pathways of RNA degradation. Cell 136: 763-776.

Ingelfinger D, Arndt-Jovin DJ, Luhrmann R, Achsel T. 2002. The human LSm1-7 proteins colocalize with the mRNA-degrading enzymes Dcp1/2 and Xrn1 in distinct cytoplasmic foci. RNA 8: 1489-1501.

Kambach C, Walke S, Young R, Avis JM, de la Fortelle E, Raker VA, Luhrmann R, Li J, Nagai K. 1999. Crystal structures of two Sm protein complexes and their implications for the assembly of the spliceosomal snRNPs. Cell 96: 375-387.

Kiefer F, Arnold K, Kunzli M, Bordoli L, Schwede T. 2009. The SWISS-MODEL Repository and associated resources. Nucleic Acids Res 37: D387-D392.

Mayes AE, Verdone L, Legrain P, Beggs JD. 1999. Characterization of Sm-like proteins in yeast and their association with U6 snRNA. EMBO J 18: 4321-4331.

Mikulecky PJ, Kaw MK, Brescia CC, Takach JC, Sledjeski DD, Feig AL. 2004. Escherichia coli Hfq has distinct interaction surfaces for DsrA, rpoS and poly(A) RNAs. Nat Struct Mol Biol 11: 1206-1214.
Mura C, Cascio D, Sawaya MR, Eisenberg DS. 2001. The crystal structure of a heptameric archaeal Sm protein: Implications for the eukaryotic snRNP core. Proc Natl Acad Sci 98: 5532-5537.

Mura C, Kozhukhovsky A, Gingery M, Phillips M, Eisenberg D. 2003a. The oligomerization and ligand-binding properties of Sm-like archaeal proteins (SmAPs). Protein Sci 12: 832-847.

Mura C, Phillips M, Kozhukhovsky A, Eisenberg D. 2003b. Structure and assembly of an augmented Sm-like archaeal protein 14-mer. Proc Natl Acad Sci 100: 4539-4544.

Naidoo N, Harrop SJ, Sobti M, Haynes PA, Szymczyna BR, Williamson JR, Curmi PM, Mabbutt BC. 2008. Crystal structure of Lsm3 octamer from Saccharomyces cerevisiae: implications for Lsm ring organisation and recruitment. J Mol Biol 377: 1357-1371.

Pilkington GR, Parker R. 2008. Pat1 contains distinct functional domains that promote P-body assembly and activation of decapping. Mol Cell Biol 28: 1298-1312.

Reijns MA, Auchynnikava T, Beggs JD. 2009. Analysis of Lsm1p and Lsm8p domains in the cellular localization of Lsm complexes in budding yeast. FEBS J 276: 3602-3617.

Salgado-Garrido J, Bragado-Nilsson E, Kandels-Lewis S, Seraphin B. 1999. Sm and Sm-like proteins assemble in two related complexes of deep evolutionary origin. EMBO J 18: 3451-3462.

Schumacher MA, Pearson RF, Moller T, Valentin-Hansen P, Brennan RG. 2002. Structures of the pleiotropic translational regulator Hfq and an Hfq-RNA complex: a bacterial Sm-like protein. EMBO J 21: $3546-3556$.

Seraphin B. 1995. Sm and Sm-like proteins belong to a large family: identification of proteins of the U6 as well as the U1, U2, U4 and U5 snRNPs. EMBO J 14: 2089-2098.

Sikorski RS, Hieter P. 1989. A system of shuttle vectors and yeast host strains designed for efficient manipulation of DNA in Saccharomyces cerevisiae. Genetics 122: 19-27.

Tharun S. 2008. Purification and analysis of the decapping activator Lsmlp-7p-Patlp complex from yeast. Methods Enzymol 448: 41-55.

Tharun S. 2009. Roles of eukaryotic Lsm proteins in the regulation of mRNA function. Int Rev Cell Mol Biol 272: 149-189.

Tharun S, He W, Mayes AE, Lennertz P, Beggs JD, Parker R. 2000. Yeast Sm-like proteins function in mRNA decapping and decay. Nature 404: 515-518.

Tharun S, Muhlrad D, Chowdhury A, Parker R. 2005. Mutations in the Saccharomyces cerevisiae LSM1 gene that affect mRNA decapping and 3' end protection. Genetics 170: 33-46.

Toro I, Thore S, Mayer C, Basquin J, Seraphin B, Suck D. 2001. RNA binding in an Sm core domain: X-ray structure and functional analysis of an archaeal Sm protein complex. EMBO J 20: 2293-2303.

Totaro A, Renzi F, La Fata G, Mattioli C, Raabe M, Urlaub H, Achsel T. 2011. The human Pat1b protein: a novel mRNA deadenylation factor identified by a new immunoprecipitation technique. Nucleic Acids Res 39: 635-647.

Vecerek B, Rajkowitsch L, Sonnleitner E, Schroeder R, Blasi U. 2008. The C-terminal domain of Escherichia coli $\mathrm{Hfq}$ is required for regulation. Nucleic Acids Res 36: 133-143.

Wilusz CJ, Wilusz J. 2005. Eukaryotic Lsm proteins: lessons from bacteria. Nat Struct Mol Biol 12: 1031-1036. 

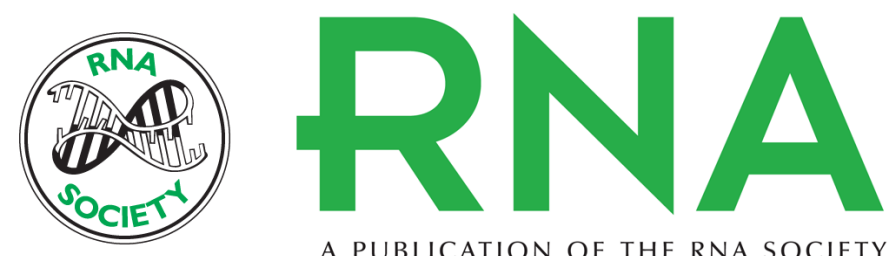

A PUBLICATION OF THE RNA SOCIETY

\section{Both Sm-domain and C-terminal extension of Lsm1 are important for the RNA-binding activity of the Lsm1-7-Pat1 complex}

Ashis Chowdhury, Kalidindi K. Raju, Swathi Kalurupalle, et al.

RNA 2012 18: 936-944 originally published online March 26, 2012

Access the most recent version at doi:10.1261/rna.029876.111

\section{Supplemental http://rnajournal.cshlp.org/content/suppl/2012/03/14/rna.029876.111.DC1 \\ Material}

References This article cites 40 articles, 17 of which can be accessed free at: http://rnajournal.cshlp.org/content/18/5/936.full.html\#ref-list-1

\section{License}

Email Alerting Receive free email alerts when new articles cite this article - sign up in the box at the Service top right corner of the article or click here.

\section{III!"II Providing Precise Solutions tor your research.}

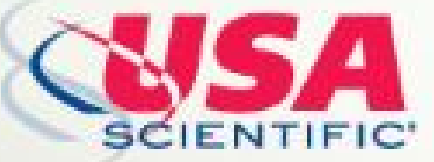

To subscribe to $R N A$ go to:

http://rnajournal.cshlp.org/subscriptions 